\title{
Inguinal abscess following Trans-abdominal Preperitoneal Mesh repair for inguinal hernia
}

\section{Rajiv Nakarmi, Muza Shrestha, Sundar Maharjan}

Department of surgery, Manmohan Memorial Medical College and Teaching Hospital, Kathmandu, Nepal.

Correspondence: Dr. Rajiv Nakarmi, Department of surgery, Manmohan Memorial Medical College and Teaching Hospital, Kathmandu, Nepal.

Email: rajivnakarmi31@gmail.com

\section{Abstract}

Introduction: Laparoscopic hernia surgery is performed by almost every general and laparoscopic surgeon worldwide, Trans-abdominal Preperitoneal (TAPP) and Totally Extraperitoneal (TEP) being the most frequently performed. Seroma formation leading to deep seated mesh infection with abscess is rare but once it occurs it can be very difficult to treat. The rate of mesh infection after open mesh repair is reported to be between $0.5 \%$ - 3\%, whereas in laparoscopic repair is less than $0.16 \%$. Seroma formation following TAPP is $3 \%-8 \%$ and following TEP is $0.5-12.2 \%$.

Case Report: A 19 years old male presented with groin swelling, fever and weight loss three months after the laparoscopic TAPP surgery for right inguinal hernia. Workup showed he had developed mesh infection with pre-peritoneal abscess which was managed with open drainage of the abscess with removal of the infected mesh.

Conclusion: Seroma formation may result in abscess formation. If occurred removal of the mesh with drainage of abscess is often required. The chance of recurrence of hernia following management of infected mesh should always be considered.

Keywords: Abscess; Hernia; Laparoscopy; Mesh infection; Trans-abdominal pre-peritoneal repair.

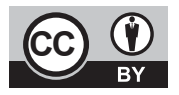

DOI: https://doi.org/10.3126/jssn.v23i1.33558

\section{Introduction}

Over the past two decades laparoscopy has been established as a superior technique in many general surgery procedures. Accordingly laparoscopic mesh repair techniques have been tried and proven to be have advantages over open hernia procedure, such as less acute and chronic pain, early return to work, with no difference in recurrence rate. ${ }^{1}$ TAPP (Trans-abdominal Pre-Peritoneal) and TEP (Totally Extraperitoneal) procedures are performed by almost every laparoscopic surgeons worldwide. It is now fast becoming a superior technique in the repair for inguinal hernia. ${ }^{2}$

The complication rates of abscess and wound infection rate is significantly low in laparoscopic approach than in open approach with similar recurrence rate. ${ }^{3,4}$ Felix et al performed laparoscopic inguinal hernia surgery in around 1000 patients and found that the complication rate Journal of Society of Surgeons of Nepal (JSSN) was $0.5 \% .{ }^{5}$ Superficial port site infections can be managed in days to weeks with appropriate antibiotics and wound care. Deep-seated mesh infection is rare but once it occurs it can be very difficult to treat and may cause chronic groin sepsis and abscess. The rate of mesh infection after open mesh repair is reported to be between $0.5 \%-3 \%$, whereas in laparoscopic repair is less than $0.16 \%{ }^{6-8}$ The patient may present with painful groin swelling, and sinus formation. ${ }^{9}$ Conservative treatment is usually not successful hence requiring removal of the mesh and drainage of collection. The mesh removal can be done by open anterior approach or by laparoscopic approach. ${ }^{10}$ Here, we present a case of abscess formation with mesh infection following TAPP for right indirect inguinal hernia which required removal of the infected mesh with drainage of abscess by anterior groin approach.

JSSN 2020; 23 (1) 


\section{Case report}

A 19 years old male presented to outpatient department with complaint of swelling in right groin noticed for four months which was gradually increasing in size. After proper assessment he was diagnosed to have complete, spontaneously reducible, right indirect inguinal hernia. Transabdominal pre-peritoneal mesh repair was done. Intraoperative findings matched clinical findings with sac extending up to the base of scrotum. The sac was completely dissected (Figure 1) and reduced and a $10 \times 15$ $\mathrm{cm}$ polypropylene micro-porous mesh was used and fixed with non-absorbable titanium tacker at cooper's ligament, lateral rectus muscle and the third one at the lateral aspect. Peritoneum was closed with continuous suture using 3-0 polyglactin. Intraoperative bleeding was minimal. Early post-operative period was uneventful and he was discharged on the second post-operative day.

He was followed up one week after for skin suture removal where he had no complaints except for some pain at the surgical site while walking. Three months after the surgery he presented in the outpatient department with complaint of mild fever and swelling at the surgery site. He also complained of decreased appetite and significant weight loss. Upon assessment it was found that he had developed an abscess at the site. Ultrasonography of the site revealed there was fluid with internal debris collection at the preperitoneal space and mesh was floating (Figure 2). Computerized tomography revealed abscess formation at the site of previous surgery at pre-peritoneal space with no intraperitoneal complications. He was admitted and started on antibiotics. Repeated ultrasound guided aspiration was not fruitful and hence open surgery with drainage of $200 \mathrm{ml}$ of pus and removal of the mesh was done. After thorough irrigation of the pre-peritoneal cavity, a drain was placed and the defect was closed using Maloney's darning technique. Post-operatively patient was kept under third generation cephalosporin and metronidazole. The patient improved and was discharged on the $7^{\text {th }}$ post-operative day.
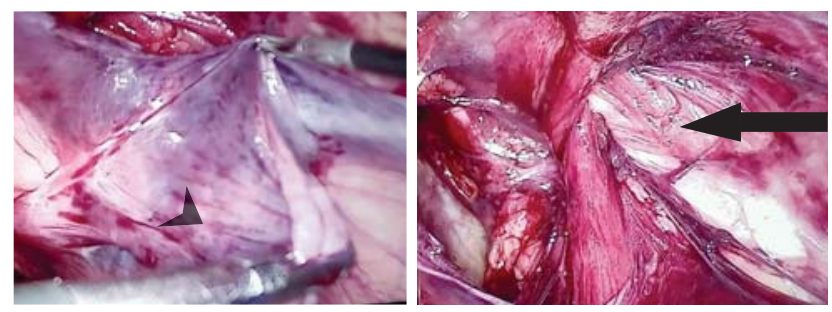

Figure 1. Intraoperative view: Arrowhead: dissected sac. Arrow: defect.

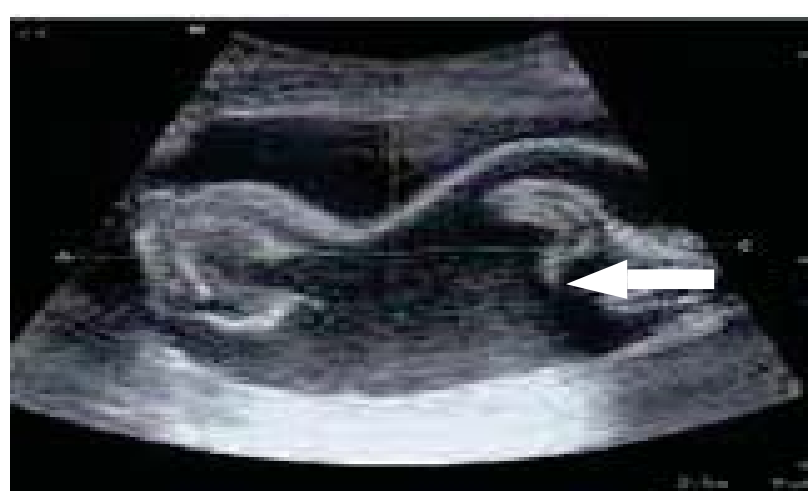

Figure 2. Ultrasonography: Arrow showing fluid collection with floating mesh

\section{Discussion}

Early superficial wound infection is related to intraoperative contamination, whereas late prosthetic infection may be associated with persistent fluid collection. ${ }^{11}$ Various factors have been instated for mesh infection that includes patient factors, technical factors and postoperative complications. Patient factors include chronic obstructive lung disease, high Body Mass Index, smoking, advance age, American Society of Anesthesiologist ASA $>3.12,13$ Technical factors include prior surgical site infections, prior surgery at the same site, iatrogenic enterotomy, microporous/ multifilament/ heavy weight/ hydrophobic mesh, vicrylprolene composite mesh, longer operating time, and lack of tissue coverage of mesh. ${ }^{12-15}$ Seroma formation following surgery is a potential risk factor for mesh infection and repeated aspiration of seroma is also regarded as one. ${ }^{10}$ The reported incidence of seroma formation after TAPP is $3.0-8.0 \%{ }^{16}$ and for TEP is $0.5-12.2 \%{ }^{17}$ The need for extensive dissection of pre-peritoneal space and placement of mesh as well as inflammatory response related to surgical applications, such as cutting, electrocautery all act for seroma formation. ${ }^{18}$ Hematoma, folded mesh can also lead to infection. ${ }^{19}$ In this patient we found that the pus collected was yellowish purulent with no evidence of hematoma. Most likely, the cause was seroma leading to infection and abscess formation as during the first surgery dissection was time consuming due to extension of hernia sac into the scrotum with defect of around $2 \mathrm{~cm}$. In an article published by Cihan in 2006, the incidence of seroma formation after laparoscopic hernia surgery in $1^{\text {st }}$ post-operative day increased from $56.7 \%$ to $66.7 \%$ after the use of ultrasonography post-operatively. ${ }^{18}$ In initial postoperative period seroma formation may only manifest as groin pain with no external features which was the scenario in our case and thus ultrasonography was not done. 
Organisms mostly associated with mesh infection are staphylococcus species mostly staphylococcus aureus, coagulase negative staphylococcus, enterococcus faecalis, Corynebacterium, pseudomonas aeruginosa and MRSA. ${ }^{20,21}$ Atypical Mycobacteria have been known to colonize tap water, natural waters, and soil and thus can easily contaminate solutions and disinfectants used in hospital settings and thus is seen in reused instruments which are not properly sterilized and not dried rapidly. ${ }^{22,23}$ Optimal sterilization of the instruments is important but if such sterilization could not be achieved due to lack of resources at least high-level disinfection has been used. $2 \%$ glutaraldehyde, $6 \%$ stabilized hydrogen peroxide and per acetic acid are frequently used disinfectants. Unlike other surgical instruments, laparoscopic instruments cannot be sterilized by autoclaving, as the high temperatures involved destroy the insulation on them. Thus, frequently used sterilization procedure has been a 20 minute exposure to $2.0-2.5 \%$ glutaraldehyde. At this current exposure time, these solutions act as high level disinfectants and not sterilants thus allowing bacterial endospores to survive. ${ }^{24}$ Current guidelines on infection control recommend a minimum exposure time of 8-12 hours to achieve the desired level of sporicidal activity of these germicides and the use of higher concentrations (3.4\%) of glutaraldehyde disinfectants. These chemicals can be used for maximum of 100 cycles or a period of 14 days $(2.5 \%$ glutaraldehyde) or 28 days (3.4\% glutaraldehyde). ${ }^{25-27}$ In our clinical setting where we reuse most of the instruments we usually use $2 \%$ glutaraldehyde and procedures done by the same instruments in other patients had not resulted in any of such complications. Atypical infection was also thought of during the management. But the culture didn't show any growth and after the mesh removal infection was controlled with third generation cephalosporin and metronidazole which was continued for 10 days postoperatively.

In this case report we have described our experience of removal of infected mesh after laparoscopic hernia surgery from anterior groin approach. The laparoscopic TAPP approach for removal of infected mesh has risk of spread/ spillage of pus in to the peritoneal cavity, adhesions. Apart from this there might be some difficulty in mesh identification due to presence of adhesions between bowel and peritoneum due to the initial surgery. ${ }^{8,28}$

Hernia recurrence after mesh removal is seen in 5\% of cases according to 40 cases review done by S Rehman et al in 2012. ${ }^{29}$ Taylor et al in 1999 reported two cases of recurrence of which one was asymptomatic. This occurred two years after the mesh removal and had to undergo open mesh repair. The patient further developed chronic groin sepsis and hence necessitated mesh removal. ${ }^{9}$ However Pradeep K et al reported the recurrence rate of $20 \%{ }^{10}$ In this case we repaired the defect with darn repair to prevent recurrence. Patient was discharged on seventh postoperative day. And was followed up on $14^{\text {th }}$ day and after two months where he had no complaints. He is planned for follow up at 1 year and 2 years.

\section{Conclusion}

Unlike superficial infection which can be treated with appropriate wound care and antibiotics, deep seated mesh infection may result in abscess formation. Mesh infection with abscess formation is rare but dreadful complication following laparoscopic hernia repair. Appropriate sterilization or high-level disinfection is a must. Postoperative pain at the surgical site should not be taken lightly and possibility of complications such as seroma, hematoma superficial surgical site infections which may lead to deep seated mesh infection can always be ruled out using ultrasonography. Once infection has developed, ultimately will require mesh removal for the optimal management. Removal of the mesh with drainage of abscess can be carried out by either laparoscopic or open approach. The chance of recurrence of hernia following management of infected mesh should always be considered.

\section{References}

1. Bullen N, Massey L, Antoniou S, Smart N, Fortelny R. Open versus laparoscopic mesh repair of primary unilateral uncomplicated inguinal hernia: a systematic review with meta-analysis and trial sequential analysis. Hernia. 2019 Jun;3(23):461-72.

2. Group TH. International guidelines for groin hernia management. Hernia. 2018 Feb;22(1):1.

3. Salvilla SA, Thusu S, Panesar SS. Analysing the benefits of laparoscopic hernia repair compared to open repair: a meta-analysis of observational studies. Journal of minimal access surgery. 2012 Oct-Dec;8(4):111.

4. Bhandarkar DS, Shankar M, Udwadia TE. Laparoscopic surgery for inguinal hernia: current status and controversies. Journal of minimal access surgery. 2006;2(3):178.

5. Felix E, Harbertson N, Vartanian S. Laparoscopic hernioplasty. Surgical endoscopy. 1999 Mar;13(4):32831. 
6. Birth M, Friedman R, Melullis M, Weiser $H$. Laparoscopic transabdominal preperitoneal hernioplasty: results of 1000 consecutive cases. Journal of laparoendoscopic surgery. 1996 Apr;6(5):293-300.

7. Litwin DE, Pham QN, Oleniuk FH, Kluftinger AM, Rossi L. Symposium on the management of inguinal hernias, 3: laparosopic groin hernia surgery: the TAPP procedure [Transabdominal preperitoneal repair]. Canadian Journal of Surgery. 1997 Jun;40(3):192.

8. Tamme C, Scheidbach H, Hampe C, Schneider C, Köckerling F. Totally extraperitoneal endoscopic inguinal hernia repair (TEP). Surgical Endoscopy and Other Interventional Techniques. 2003 Feb;17(2):1905 .

9. Taylor S, O'dwyer P. Chronic groin sepsis following tension-free inguinal hernioplasty. British journal of surgery. 1999 Apr;86(4):562-5.

10. Chowbey PK, Khullar R, Sharma A, Soni V, Baijal M, Garg N, et al. Laparoscopic management of infected mesh after laparoscopic inguinal hernia repair. Surgical Laparoscopy, Endoscopy \& Percutaneous Techniques. 2015 Apr;25(2):125-8.

11. Mann DV, Prout J, Havranek E, Gould S, Darzi A. Late-onset deep prosthetic infection following mesh repair of inguinal hernia. The American journal of surgery. 1998 Jul;176(1):12-4.

12. Sanchez VM, Abi-Haidar YE, Itani KM. Mesh infection in ventral incisional hernia repair: incidence, contributing factors, and treatment. Surgical infections. 2011 Aug;12(3):205-10.

13. Mavros MN, Athanasiou S, Alexiou VG, Mitsikostas PK, Peppas G, Falagas ME. Risk factors for meshrelated infections after hernia repair surgery: a metaanalysis of cohort studies. World journal of surgery. 2011 Sep;35(11):2389.

14. Harrell A, Novitsky Y, Kercher K, Foster M, Burns J, Kuwada T, et al. In vitro infectability of prosthetic mesh by methicillin-resistant Staphylococcus aureus. Hernia. 2006 Apr;10(2):120-4.

15. Halaweish I, Harth K, Broome A-M, Voskerician G, Jacobs MR, Rosen MJ. Novel in vitro model for assessing susceptibility of synthetic hernia repair meshes to Staphylococcus aureus infection using green fluorescent protein-labeled bacteria and modern imaging techniques. Surgical infections. 2010 Oct;11(5):449-54.
16. Reddy V, Sutton C, Bloxham L, Garcea G, Ubhi S, Robertson G. Laparoscopic repair of direct inguinal hernia: a new technique that reduces the development of postoperative seroma. Hernia. 2007 Nov;11(5):3936.

17. Berney C. The Endoloop technique for the primary closure of direct inguinal hernia defect during the endoscopic totally extraperitoneal approach. Hernia. 2012 Jun;16(3):301-5.

18. Cihan A, Ozdemir H, Uçan B, Acun Z, Comert M, Tascilar O, et al. Fade or fate. Seroma in laparoscopic inguinal hernia repair. Surgical endoscopy. 2006 Feb;20(2):325-8.

19. Foschi D, Corsi F, Cellerino P, Trabucchi A, Trabucchi E. Late rejection of the mesh after laparoscopic hernia repair. Surgical endoscopy. 1998 Apr;12(5):455-7.

20. Delikoukos S, Tzovaras G, Liakou P, Mantzos F, Hatzitheofilou C. Late-onset deep mesh infection after inguinal hernia repair. Hernia. 2007 Feb;11(1):15-7.

21. Stremitzer S, Bachleitner-Hofmann T, Gradl B, Gruenbeck M, Bachleitner-Hofmann B, Mittlboeck $\mathrm{M}$, et al. Mesh graft infection following abdominal hernia repair: risk factor evaluation and strategies of mesh graft preservation. A retrospective analysis of 476 operations. World journal of surgery. 2010 Apr;34(7):1702-9.

22. Sohail MR, Smilack JD. Hernia repair mesh-associated Mycobacterium goodii infection. Journal of clinical microbiology. 2004 Jun;42(6):2858-60.

23. Devi DG, Sridaran D, Indumathi V, Babu P, Belwadi M, Swamy A. Isolation of Mycobacterium Chelonae from wound infection following laparoscopy: a case report. Indian J Tuberculosis. 2004 Jan;51(3):149-51.

24. Ramesh H, Prakash K, Lekha V, Jacob G, Venugopal A, Venugopal B. Port-site tuberculosis after laparoscopy. Surgical endoscopy. 2003 Jul;17(6):930.

25. Rutala WA, Weber DJ. Disinfection and sterilization in health care facilities: what clinicians need to know. Clinical infectious diseases. 2004 Sep;39(5):702-9.

26. Leid JG, Shirtliff ME, Costerton JW, Stoodley P. Human leukocytes adhere to, penetrate, and respond to Staphylococcus aureus biofilms. Infection and immunity. 2002 Nov;70(11):6339-45. 
27. Chaudhuri S, Sarkar D, Mukerji R. Diagnosis and management of atypical mycobacterial infection after laparoscopic surgery. Indian Journal of Surgery. 2010 Dec;72(6):438-42.

28. Chihara N, Mishima K, Suzuki H, Watanabe M, Toyoda T, Uchida E. Totally extraperitoneal (TEP) removal of an infected mesh by laparoscopy after open preperitoneal repair: initial case report. Journal of Nippon Medical School. 2017 Jan;84(1):45-8.

29. Rehman S, Khan S, Pervaiz A, Perry E. Recurrence of inguinal herniae following removal of infected prosthetic meshes: a review of the literature. Hernia. 2012 Apr;16(2):123-6. 